\title{
CALCIFILAXIS COMO COMPLICACIÓN CUTÁNEA EN PACIENTE CON INSUFICIENCIA RENAL CRÓNICA EN DIÁLISIS
}

\author{
Liliana Herrera', Carlos Avellaneda², Norbey Tique ${ }^{3}$ \\ ${ }^{1}$ Especialista en Dermatología. Hospital Militar Central. Universidad Militar Nueva Granada. \\ ${ }^{2}$ Especialista en Dermatología. Hospital Militar Central. Universidad Militar Nueva Granada. \\ ${ }^{3}$ Residente III año. Hospital Militar Central. Universidad Militar Nueva Granada.
}

Correspondencia:mdtique@gmail.com

Recibido: Marzo 5 de 2013 Aceptado: Julio 24 de 2013

\begin{abstract}
Resumen
La calcifilaxis generalmente hace referencia a una enfermedad caracterizada por lesiones violáceas reticuladas dolorosas que progresan a necrosis, de predominio en miembros inferiores, en pacientes con falla renal especialmente que se encuentran en manejo con hemodiálisis, tiene una mortalidad que en algunos estudios alcanza hasta el $80 \%$. Hay una mayor incidencia de eventos cardiovasculares en estos pacientes. Presentamos el caso de una paciente con calcifilaxis secundaria a insuficiencia renal crónica, en la que se resalta el alto grado de mortalidad de la enfermedad.
\end{abstract}

Palabras clave: Calcifilaxis, falla renal, calcificación metastásica.

\section{CALCIPHYLAXIS SKIN AS A COMPLICATION IN PATIENTS WITH CHRONIC RENAL DIALYSIS}

\begin{abstract}
Calciphylaxis usually refers to a disease characterized by reticulated violet lesions painful to touch which progress to necrosis and predominate in lower extremities in patients diagnosed with renal failure and who are being managed with hemodialysis. This disease has a high mortality rate that in some studies reaches up to $80 \%$. There is an increase in cardiovascular events in these patients. We present the case of a patient who is diagnosed with calciphylaxis secondary to chronic renal failure, in which we highlight the elevated rate of mortality secondary to this disease.
\end{abstract}

Key words: Calciphylaxis, renal failure, metastatic calcification.

\section{CALCIFILAXIA COMO COMPLICAÇÃO CUTÂNEA EM PACIENTE COM INSUFICIÊNCIA RENAL CRÔNICA EM DIÁLISE}

\section{Resumo}

A calcifilaxia geralmente faz referência a uma doença caracterizada por lesões violáceas reticuladas dolorosas que progridem a necrose, de predomínio em membros inferiores, em pacientes com falha renal especialmente que se encontram em manejo com hemodiálise, tem uma mortalidade que em alguns estudos atinge até o $80 \%$. Há uma maior incidência de eventos cardiovasculares em estes pacientes. Apresentamos o caso de uma paciente com calcifilaxia secundaria a insuficiência renal crônica, na que se resalta a alta série de mortalidade da doença.

Palavras chave: Calcifilaxia, falha renal, calcificação metastática. 


\section{Introducción}

La calcifilaxis es una condición poco frecuente pero devastadora que puede llevar a un compromiso no solo de la piel sino de músculo, fascia y órganos internos, causando isquemia y necrosis de estos tejidos por depósito de calcio en los vasos de pequeño y mediano calibre (1).

Esta condición afecta principalmente a pacientes con falla renal crónica, manejados con hemodiálisis, y luego de trasplantes renales; otras condiciones como hiperparatiroidismo primario, y menos frecuente como malignidad también ha sido reportada. La calcifilaxis está relacionada con una alta mortalidad con un rango que oscila entre 46 y $80 \%$, y también con sobreinfección que puede llevar a sepsis.

\section{Reporte de caso}

Paciente de 61 años quien es hospitalizada por cuadro clínico con una semana de evolución, que consiste en aparición de lesiones eritematosas, dolorosas, en miembros inferiores, de crecimiento progresivo. Éstas iniciaron como placas y algunas evolucionaron hacia nódulos, posteriormente se presenta ulceración central. No refiere fiebre.

Esta paciente tiene antecedentes de falla renal crónica secundaria a hipertensión arterial desde hace cinco años, actualmente en manejo con diálisis tipo hemodiálisis, también diabetes mellitus en tratamiento, e hiperparatiroidismo secundario. En el examen físico se encuentra en buen estado general, hemodinámicamente estable, presenta en miembros inferiores múltiples placas y nódulos eritematosos algunos con ulceración central y costra serohemática, de bordes mal definidos, y dolorosas a la palpación. Pulsos poplíteos, pedios positivos (figura 1). Es valorada por el servicio de dermatología quien propone como diagnóstico presuntivo una calcifilaxis como consecuencia de la insuficiencia renal.

Se realiza una biopsia de lesiones, paraclínicos que incluyen pruebas de función renal, niveles sanguíneos de calcio, fosfato, paratohormona, hemograma completo, proteína $\mathrm{C}$ reactiva, velocidad de sedimentación globular. Además, se recomendó manejo adecuado de analgesia, desbridamiento de tejido necrótico en lesiones, y manejo con diálisis según criterio de nefrología. El reporte de la biopsia de piel evidencia depósito de calcio en pared de vasos a nivel de la dermis (figura 2).
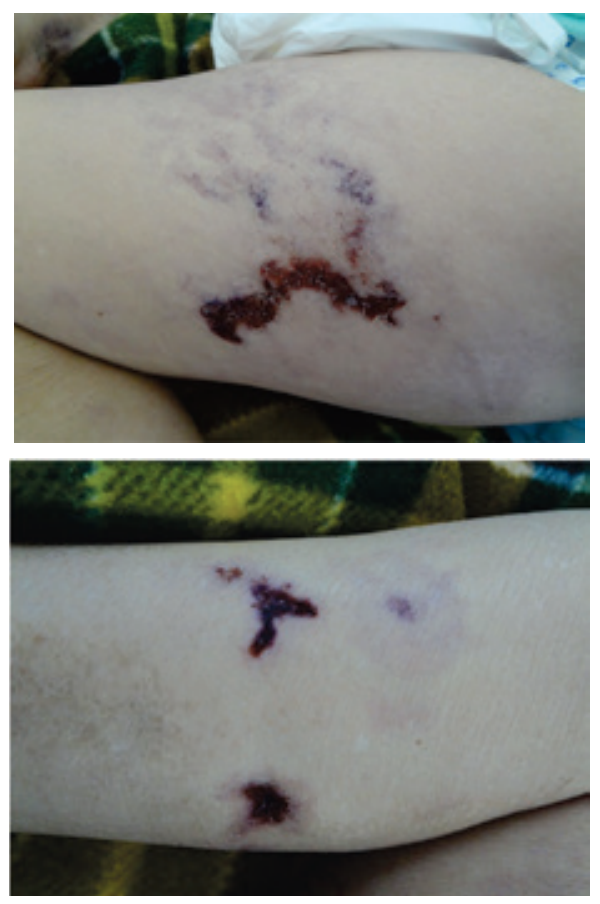

Figura 1. A: Placas eritematosas y violáceas de bordes isquémicos mal definidos, algunas con costra serohemáticas en región externa de muslo izquierdo. B: Placas con centro necrótico y nódulos eritematosos en región lateral de pierna
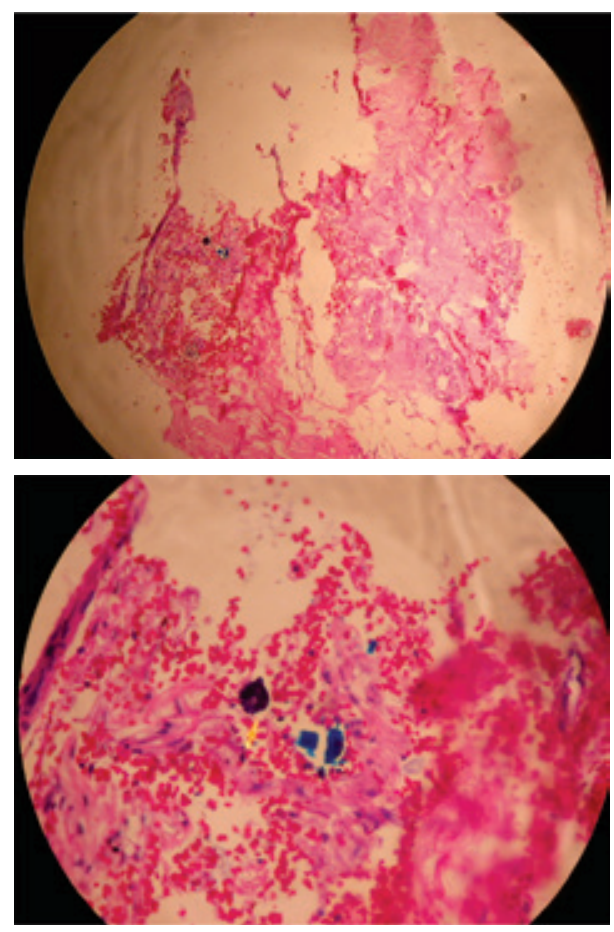

Figura 2. A: Histopatología evidencia depósito de calcio (flecha amarilla) en pared de vasos a nivel de la dermis B: Vista a mayor aumento. 
La paciente reingresa diez días después por cuadro de 3 días de evolución de dolor en miembro superior izquierdo, asociado a cianosis distal y se documenta paraclínicamente infarto agudo de miocardio sin elevación de ST, con trombosis a nivel de la arteria subclavia izquierda. En el examen físico se encuentra consciente, hemodinámicamente estable, en miembros superior izquierdo cianosis, frialdad, lívido reticularis, necrosis distal en cuarto y quinto dedos, sin signos de SIRS. En miembros inferiores progresión de placas con áreas de necrosis, bordes bien definidos, sin signos de sobreinfección (figura 3).
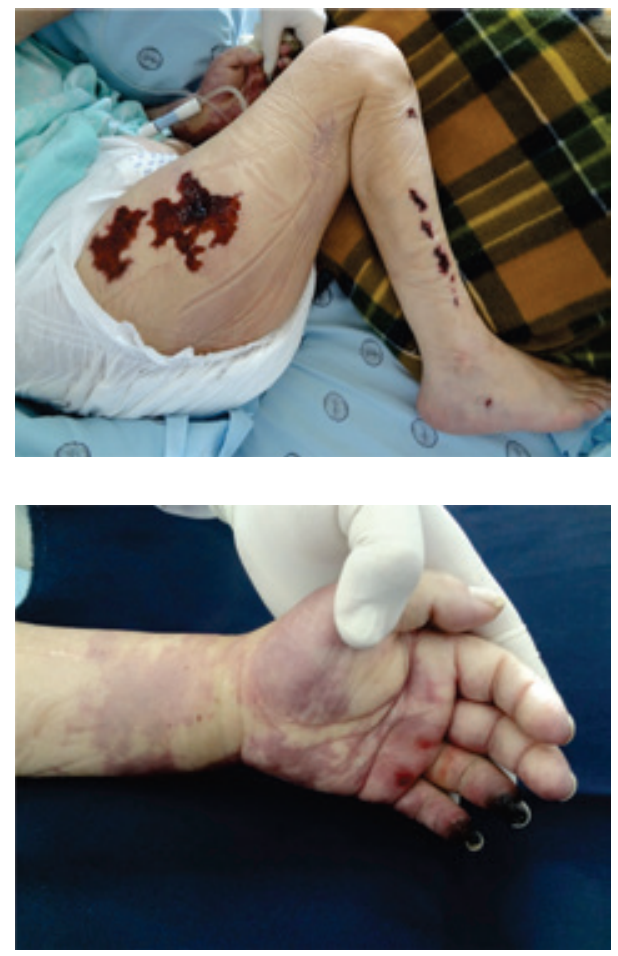

Figura 3. A: Progresión de lesiones isquémicas en miembros inferiores. B: Cianosis en miembro superior izquierdo con necrosis distal de cuarto y quinto dedo.

Se considera que la paciente no es candidata a revascularización por el grado de necrosis de tejidos distales, y alto riesgo de síndrome de revascularización con alta mortalidad, la posibilidad de manejo quirúrgico es diferida por el uso concomitante de asa, y clopidrogel. Evoluciona hacia el deterioro, con compromiso del estado de conciencia, hipotensión persistente, dificultad respiratoria, edema progresivo en miembros inferiores y fallece siete días luego de ingreso.

\section{Revisión}

Se conoce que el primer reporte de calcifilaxis fue hecho en 1898 por Bryant y White, ellos hicieron referencia a la relación entre gangrena cutánea y calcificación (2).

El término calcifilaxis fue acuñado por Selye en 1962 como una condición de hipersensibilidad sistémica desencadenada por un agente sensibilizante, lo que originaba una calcificación metastásica en diferentes órganos; algo similar a una anafilaxia, ésto fue conceptualizado luego de varios trabajos experimentales en animales. Años más tarde se describió un síndrome caracterizado por isquemia y necrosis de tejido periférico, y úlceras cutáneas en pacientes urémicos, y debido a la semejanza con el modelo de Selye fue llamado calcifilaxis.

Generalmente el término calcifilaxis hace referencia al cuadro clínico caracterizado por lesiones violáceas reticuladas dolorosas que progresan a necrosis, que típicamente compromete a las extremidades inferiores, en pacientes con falla renal especialmente los que están en manejo con hemodiálisis, con elevación de la hormona paratiroidea, con alteración del metabolismo de calcio y fósforo y que con frecuencia tiene un desenlace fatal. En otras enfermedades (neoplasias, autoinmunes, inflamatorias) que cursan con calcificación en la pared de los vasos, pero que no ocurre en pacientes con enfermedad renal, ni con las otras características descritas, también se ha utilizado el término de calcifilaxis.

La patogénesis de esta enfermedad no es del todo clara. Uno de los factores principalmente implicados en la mayoría de los pacientes, es la alteración en el metabolismo del calcio y el fosfato; como consecuencia de la falla renal y el hiperparatiroidismo secundario. A pesar de que estas alteraciones en el metabolismo se consideran una condición sine qua non de calcifilaxis, reportes recientes han demostrado evidencia de casos con niveles de calcio y fósforo normales $(3,4)$. Recientes estudios han revelado la presencia de glicoproteínas óseas como proteína de matriz Gla, osteopontina, proteína morfogénica de hueso en arterias calcificadas patológicamente, lo que sugiere un rol en la calcificación vascular $(5,6)$.

Otras condiciones también se han relacionado con calcifilaxis como hiperparatiroidismo primario, malignidad que incluyen melanoma, cáncer metastásico de mama, mieloma múltiples y leucemias. Enfermedades 
sistémicas como lupus eritematoso sistémico, artritis reumatoide, enfermedad de crohn's, enfermedad hepática alcohólica, cirrosis hepática autoinmune, estados de hipercoagulabilidad como deficiencia de proteína C y S, síndrome antifosfolipido y obesidad. También algunos fármacos son reportados como glucocorticoides, dosis altas de vitamina $\mathrm{D}$ y sus análogos, warfarina y agentes quimiotácticos (2).

Las manifestaciones clínicas están dadas por los hallazgos cutáneos y aquellos que son sistémicos. Dentro de los primeros incluye como lesión inicial máculas eritematosas o violáceas, dolorosas, que pueden semejar livedo reticularis y raramente ampollas flácidas y hemorrágicas, éstas evolucionan a placas o nódulos subcutáneos eritematosos y periferia violácea que luego hacen necrosis central, y pueden ulcerarse. Estas lesiones tienen predilección por áreas con alto contenido de tejido adiposo, siendo más frecuentes sobre muslos, piernas, glúteos, parte inferior del abdomen, también en dedos de manos y pies, senos, lengua y pene. Las lesiones tienden a ser simétricas, bilaterales y bien demarcadas; es característica en toda la evolución la presencia de dolor, que puede llegar a requerir manejo con narcóticos (7). Son menos frecuentes las gangrenas periféricas de los dedos de pies y manos.

Las úlceras con tejido necrótico tienen alto riesgo de sobreinfección, y en el ámbito intrahospitalario el porcentaje de colonización por Pseudomona es del $50 \%$, por lo que debe tenerse en cuenta cuando el paciente se encuentra en este medio (8). Se ha referido que el patrón de distribución podría predecir el pronóstico, siendo éste mejor para las lesiones distales o acral (dedos de manos, pies y pene), que las que involucran segmentos proximales como muslos, glúteos, abdomen y tronco.

Las manifestaciones sistémicas están en relación con calcificación vascular y extravascular de tejidos blandos y órganos internos, y muchos de éstos también pueden ser explicados por otras patologías frecuentes que suelen asociarse con la enfermedad renal crónica. Estas manifestaciones incluyen, debilidad muscular, miositis severa, embolia cerebral calcificada, infarto cerebral, demencia, calcificaciones metastásicas en pulmón con insuficiencia respiratoria aguda, compromiso cardiaco por calcificaciones, con infarto, disfunción ventricular, calcificaciones del sistema de conducción que ha sido llamado por algunos "corazón óseo", calcificaciones de estómago y ocasionalmente de páncreas y glándulas suprarrenales.

El paciente con insuficiencia renal crónica tiene mayor probabilidad de hacer eventos coronarios agudos; y el grado de excreción de albumina es un marcador de riesgo cardiovascular en pacientes diabéticos como en los no diabéticos. Se ha encontrado que el $40 \%$ de los pacientes con insuficiencia renal crónica con una tasa media de filtración glomerular de $33 \mathrm{ml} / \mathrm{min}$ muestran calcificaciones coronarias, frente al $13 \%$ de los controles (9).

Los hallazgos de laboratorio en casos típicos de calcifilaxis asociados a falla renal son elevación de hormona paratiroidea, niveles séricos de calcio, fosfato, y elevación del producto calcio fósforo. Elevación de fosfatasa alcalina, creatinina y anemia. Sin embargo, valores normales de estos parámetros no excluyen el diagnóstico de calcifilaxis.

En lesiones sugestivas de sobreinfección se debe tomar cultivo de secreción, hemograma completo, velocidad de sedimentación globular, y proteína $\mathrm{C}$ reactiva. La radiografía puede evidenciar calcificaciones, en el contexto del paciente con calcifilaxis, pero su utilidad es limitada. La ultrasonografía de alta resolución puede ayudar en el diagnóstico de las lesiones, antes de que ocurra las lesiones cutáneas típicas.

Los hallazgos histopatológicos que definen la calcifilaxis son la presencia de calcificaciones en vasos de pequeño y mediano calibre que se encuentran en la dermis y la grasa subcutánea. El depósito de calcio suele ser extenso, concéntrico en la pared del vaso y con un patrón con forma de anillo. Éstos se hallan principalmente entre la media y la intima. La tinción de hematoxilina y eosina generalmente es suficiente para evidenciar estos hallazgos, y en pocas ocasiones se requiere tinción especial de von Kossa que pone de realce los depósitos de calcio. Se ha propuesto la fibrosis endovascular como mecanismo activo de la isquemia.

Algunas veces las calcificaciones pueden estar asociadas a trombosis luminal. Otros hallazgos incluyen ulceración secundaria por la isquemia, necrosis epidérmica, dérmica y subcutánea, degeneración y necrosis de colágeno dérmico. Extravasación de eritrocitos en dermis y subcutis es un evento temprano. 
Esta condición se ha asociado con una alta mortalidad con un rango entre 46 y $80 \%(1,10)$. La principal causa es sepsis en relación con la sobre infección de ulceras con tejido necrótico. Varios estudios han encontrado que la ubicación proximal de las lesiones conlleva a peor pronóstico que las distales. Otros factores de mal pronóstico incluyen intervención tardía, lesiones en piel que comprometen el tronco, ulceración de las lesiones, sexo femenino, incremento de peso, y necesidad de intervención quirúrgica vascular.

El tratamiento de la calcifilaxis debe ser guiado por un equipo multidisciplinario. Se debe evaluar y buscar el control de todos los factores desencadenantes y agravantes como insuficiencia renal, diabetes mellitus, hipertensión arterial, artritis reumatoide, Lupus Eritematoso Sistémico, síndrome antifosfolípido, suplementos de vitamina $\mathrm{D}$, trauma local, fármacos inmunosupresores. Hay reportes que los esteroides mejoran las lesiones no ulceradas de pacientes con calcifilaxis (11). La detección temprana es la medida que ha mostrado que mejora el pronóstico.

El enfoque terapéutico está dirigido a:

- Mantener un equilibrio adecuado en los niveles séricos de calcio y fosfato; como control del hipoparatiroidismo secundario.

- El etidronato disódico, y pamidronato tienen un papel importante ya que impiden la calcificación en la pared de los vasos (12), otros medicamentos también reportados con eficacia son el Cinacalcet y tiosulfato de sodio.

- En cuanto a la paratiroidectomia, no hay indicaciones claras en calcifilaxis, su uso ha sido controversial. Hay estudios que la relacionan con mayor cicatrización de heridas, disminución de tejido necrótico, disminución del dolor y una mayor sobre vida $(13,14)$.

- Manejo de las heridas, lo que indica desbridamiento quirúrgico de tejido necrótico, uso de hidrocoliodes cuando sea necesario, manejo temprano de sobreinfecciones. Esto último teniendo en cuenta que la mortalidad se relacione con esta causa.

- Manejo del dolor.

\section{Conclusión}

La calcifilaxis es una enfermedad que con frecuencia se observa en pacientes con insuficiencia renal crónica que se encuentran en hemodiálisis, fisiopatológicamente caracterizada por depósitos de calcio en la capa media de las paredes de pequeñas y medianas arterias de la dermis; como consecuencia de la alteración en el metabolismo del calcio y fósforo. Los pacientes tienen un aumento del riesgo de eventos cardiovasculares isquémicos, en relación con calcificaciones en arterias coronarias y válvulas cardiacas. La mortalidad alcanza el $80 \%$ principalmente asociado con sepsis por sobreinfección de lesiones en piel.

\section{Referencias}

1. Ng AT, Peng DH. Calciphylaxis. Dermatologic therapy. 2011; 24: 256-262.

2. Daudén E, Oñate MJ. Calciphylaxis. Dermatol Clin. 2008; 26: 557- 568.

3. Budisavljevic MN, Cheek D, Poth DW. Calciphylaxis in chronic renal failure. J Am Soc Nephrol. 1996; 7: 978-82.

4. Smiley CM, Hanlon SU, Michel DM. Calciphylaxis in moderate insufficiency: changing disease concepts. Am J Nephrol. 2000; 20: 324-8.

5. Canfield AE, Farrington C, Dziobon MD, Boot-Handford RP, Heagerty AM, Kumar SN, et. al. The involvement of matrix glycoproteins in vascular calcification and fibrosis: an immunohistochemical study. J Pathol. 2002; 196: 228-34.

6. Griethe W, Schmitt R, Jurgensen JS, Bachmann S, Eckardt KU, Schindler R. Bone morphogenic protein-4 expression in vascular lesions of calciphylaxis. J Nephrol. 2003; 16: 728-32.

7. Boccaletti VP, Ricci R, Sebastio N, Cortellini P, Alinovi A. Penile calciphylaxis. Arch Dermatol. 2000; 136: 259-64.

8. López F, San Juan R, Díaz C. Infecciones por pseudomonas y otros bacilos gramnegativos no fermentadores. Medicine. 2006; 9(52): 3387-3397.

9. González M, Casanova R, Cervantes C, García A, Peraira J, Prieto E, et al. Enfermedad cardiovascular y función renal. Mecanismos patogénicos. Rev Esp Cardiol Supl. 2008; 8:10E21E.

10. Weenig RH, Sewell LD, Davis MD, McCarthy JT, Pittelkow MR. Calciphylaxis: natural history, risk factor analysis, and outcome. J am Acad Dermatol. 2007; 56: 569-679.

11. Fine A, Zacharias J. Calciphylaxis is usually nonulcerating: risk factors, outcome and therapy. Kidney Int. 2002; 61: 2210-7.

12. Monney P, Nguyen QV, Perroud H, Descombes E. Rapid improvement of calciphylaxis after intravenous pamidronate therapy in a patient with chronic renal failure. Nephrol Dial Transplant. 2004; 19: 2130-2.

13. Duffy A, Schurr M, Warner T, Chen H. Long-term outcomes in patients with calciphylaxis from hyperparathyroidism. Ann Surg Oncol. 2006; 13: 96-102.

14. Couto FM, Chen H, Blank RD, Drezner MK. Calciphylaxis in the absence of end-stage renal disease. Endocr Pract. 2006; 12: 406-10. 